\title{
Metabolic Regulation of Cell Differentiation
}

\author{
Elizabeth Frayne \\ College of General Studies, University of Phoenix, USA \\ Email: efrayne@verizon.net
}

Received: 20 July 2020; Revised: 3 September 2020; Accepted: 19 October 2020

\begin{abstract}
Growing research suggests that metabolism itself may play a role in regulating cellular differentiation. This concept stems from three different experimental approaches involving hypoxia, nutrient metabolites, and phosphate analogues, that appear to modulate cellular differentiation by influencing the available cellular energy needed for biosynthetic processes. Possible explanatory mechanisms that could lead to the integration of changes in metabolism with transcriptional regulation include the availability of key metabolites needed for epigenetic changes to occur and/or kinases that can sense the cellular energy charge.
\end{abstract}

Keywords: cell differentiation, adenylate energy charge, phosphate analogues

\section{Introduction}

Current research raises the question of whether metabolic activity is a cause or a consequence of cell differentiation [1-2]. Clearly cell differentiation results from cell specialization and the synthesis of specific macromolecules such as proteins, all of which requires energy. Generally, there is an increase in mRNA and concomitant protein synthesis via enhanced transcription, stabilization of mRNA, and/or the translation of stored mRNAs. We know that signaling mechanisms or cues such as growth factors, retinoic acid, and cytoplasmic determinants, as well as asymmetric cell division, play a role in these processes [3]. Also, epigenetic changes are known to be involved in regulating the decision to adopt a cell fate, such as deciding between a stem cell, progenitor, or a mature cell fate [4]. Less clear, however, is the role of metabolism and the energy charge in the cell during cellular differentiation. Increasing evidence suggests that the energy associated with metabolism may be more than merely a requirement for the steps leading to cell differentiation and may instead influence the degree of differentiation as well as specific decisions related to cell fate, function, and/ or morphology. Albeit some steps may not require energy per se, but the primary outcome may be determined by the energy state.

\section{Cellular energy charge}

There are many steps during cell differentiation that do not require cell or tissue specific proteins but rather require more general housekeeping proteins. These steps include changes in cell shape involving cytoskeletal proteins, energy production via the Krebs's cycle and mitochondrial proteins, and basic biosynthetic activity involving RNA polymerases, ribosomal proteins, etc. All these processes must be coordinated with the available energy and indeed appear to respond

Copyright (C2020 Elizabeth Frayne.

DOI: https://doi.org/10.37256/acbr.112022543

This is an open-access article distributed under a CC BY license

(Creative Commons Attribution 4.0 International License)

https://creativecommons.org/licenses/by/4.0/ 
to changes in the cellular energy charge (AMP/ATP and ADP/ATP ratios). For instance, platelet cell aggregation appears to respond to changes in the adenylate energy charge (AEC) rather than total ATP levels [5]. Also, biosynthetic enzymes are well known to be regulated by AEC [6]. Adenosine nucleotides are not only participants in such metabolic reactions, but they also act in the allosteric control of numerous key regulatory enzymes in biosynthetic pathways. When the energy charge increases from 0.6 to 1.0, enzymes controlling anabolic pathways are activated such as citrate lyase and phosphoribosyl pyrophosphate (PRPP) synthetase. In mammalian cells, AMP-activated protein kinase (AMPK) senses when the cell's energy charge is low and switches on energy-producing pathways, while switching off energy-consuming pathways to restore cellular ATP levels [7]. This kinase works in part by phosphorylating metabolic enzymes such as, Acetyl-CoA carboxylase to turn off fatty acid synthesis, as well as transcriptional regulators such as SREBPS (sterol regulatory element-binding proteins) to turn off sterol synthesis. It can also turn off protein synthesis by phosphorylating proteins involved in the regulation of mammalian target of rapamycin complex 1 (mTORC1).

\section{Hypoxia}

Some studies using hypoxic conditions suggest that the cellular energy charge controls more than basic metabolism by influencing the degree of cellular differentiation and/or cell specific specialization. Restricting, the availability of oxygen is a way to influence the AEC via oxidative phosphorylation [8]. An interesting example of this is seen in studies of induced pluripotent stems cells. When somatic cells are genetically re-programed to become stem cells, cells go from a state of high oxidative phosphorylation to a state of low oxidative phosphorylation and rely instead on glycolysis [2, 9]. Interestingly, this process can be accelerated by restricting the available oxygen, and presumably the energy charge, which then promotes stem cell formation [10].

\section{Energy rich metabolites}

Another approach to manipulate energy metabolism during differentiation involves the addition of energy rich metabolites to culture media, such as during the study of neural stem cell differentiation [11]. When the neuronal culture medium is supplemented with a mixture composed of branched-chain amino acids, essential amino acids, TCA cycle precursors and co-factors, the number and characteristic features of neuronal dendrites is increased. The nutrient rich conditions drive mitochondrial remodeling with a resultant increase in cellular ATP that leads to the activation of mTORC1 dependent anabolism. Furthermore, the addition of fatty acids to induced pluripotent cardiomyocytes helps to promote their differentiation into more mature cardiomyocytes [12]. Note, cardiomyocytes are well known to prefer fatty acids as substrates for cellular energy.

\section{Phosphate analogues}

More recently my own research has found that use of the phosphate analogue, thio-phosphate, during cell culture appears to result in an increase in the cellular energy charge [1]. This analogue reduces RNA turnover by creating phosphorothioate linkages in RNA that inhibit cellular nucleases, which then allows the accumulation of significant amounts of mRNA and other non-ribosomal RNA in the cell. The energy savings from reduced RNA turnover results in an enhanced biosynthetic profile, the result of transcriptional changes rather than differential mRNA stabilization. In E coli, RNA seq studies of the analogue show enhancements in the transcription of genes for RNA processing enzymes, ribosomal proteins, tRNA charging enzymes, translational proteins, as well as outer membrane, plasma membrane, and periplasmic proteins. There are also increases in transcripts for genes involved in the synthesis of amino acids, fatty acids, carbohydrates, cell structures, cofactors, vitamins, and secondary metabolites. None of these changes correlate mRNA stability, which suggests that transcriptional control mechanisms are at play, in response to the presumed increase in cellular energy. Additional evidence comes from changes in cells observed at the protein level, such as in yeast where thio-phosphate enhances total protein secretion and in HEK293 cells where it appears to enhance the expression of neurofilaments [13]. 
In E. coli dramatic morphological changes are also observed using thio-phosphate instead of phosphate in culture media [1]. Namely, the bacteria form extensive filaments. Prolonged aggregation of flocculant yeast has also been observed (unpublished observations). In the case of Brewer's yeast, the stabilizing impact on mRNA levels may be the cause of this temporary effect, that then changes with continued growth. However, in E coli it appears the presumed increased energy charge results in a new altered morphological state. This suggests that an increase in the cellular energy charge can promote cell specific states. It will be interesting to examine the impact of thio-phosphate in models of cell differentiation in eukaryotes.

\section{Possible mechanisms}

If the energy charge in the cell does play a role in cell differentiation and/or cell specification, this raises the question of how this might occur. Both eukaryotes and bacteria methylate DNA but bacteria unlike eukaryotes methylate adenine residues in DNA rather than cytosine [14]. Nevertheless, such epigenetic changes could be a possible mechanism. In eukaryotic cells mitochondria may be an important intermediary in this process. It has been suggested that the availability of mitochondrial metabolites such as acetyl-CoA, citrate, and alpha-ketoglutarate involved in histone acetylation and methylation, may impact epigenetic processes that could then modulate gene expression and stem cell decisions $[2,9]$.

Another likely mechanism by which the energy could regulate transcription is via the phosphorylation of regulatory proteins. While phosphorylation of ser, thr, and try amino acids in bacteria is rare, it does occur [15]. In addition, recent studies indicate that $10 \%$ of his residues in the E coli proteome are phosphorylated [16]. So, there could be another kinase much like AMPK that is able to sense the energy charge and respond accordingly. Phosphorylation of sigma factors could certainly result in transcriptional changes. In fact, recent studies using Vibrio parahaemolyticus indicate transcriptional regulation via phosphorylation of inactive sigma factors enables them to interact with the beta' subunit of RNA polymerase [17].

\section{References}

[1] Frayne E. Global profile changes in transcripts induced with a phosphate analogue: implications for gene regulation. Mol Cell Biochem. 2020; 468: 111-120. Available from: doi:10.1007/s11010-020-03715-9.

[2] Teslaa T, Teitell M. Pluripotent stem cell energy metabolism: an update. The EMBO Journal. 2015; 34(2): 138-153. Available from: doi:10.15252/embj.201490446.

[3] Ali D, Abuelreich S, Alkeraishan N, Bin Shwish N, Hamam R, Kassem M. Multiple intracellular signaling pathways orchestrate adipocytic differentiation of human bone marrow stromal stem cells. Biosci Rep. 2018; 38(1): BSR20171252. Available from: doi:10.1042/BSR20171252.

[4] Pérez-Campo F, Riancho J. Epigenetic mechanisms regulating mesenchymal stem cell differentiation. Current genomics. 2015; 16(6): 368-383. Available from: doi:10.2174/1389202916666150817202559.

[5] Dangelmaier C, Holmsen H. Glyoxylate lowers metabolic ATP in human platelets without altering adenylate energy charge or aggregation. Platelets. 2014; 25(1): 36-44. Available from: doi:org/10.3109/09537104.2013.775571.

[6] D E Atkinson. The energy charge of the adenylate pool as a regulatory parameter. Interaction with feedback modifers. Biochemistry. 1968; 7(11): 4030-4034.

[7] Lin S-C, Hardie D. AMPK: Sensing glucose as well as cellular energy status. Cell Metabolism. 2018; 27(2): 299313. Available from: doi:10.1016/j.cmet.2017.10.009.

[8] Berg JM, Tymoczko JL, Stryer L. The regulation of cellular respiration is governed primarily by the need for ATP. Biochemistry. 5th edition. New York: W H Freeman; 2002. Available from: https://www.ncbi.nlm.nih.gov/books/ NBK22448/.

[9] Lisowski P, Kannan P, Mlody B, Prigione A. Mitochondria and the dynamic control of stem cell homeostasis. EMBO Reports. 2018; 19(5): e45432. Available from: https://doi.org/10.15252/embr.201745432.

[10] Hawkins KE, Sharp TV, McKay TR. The role of hypoxia in stem cell potency and differentiation. Regenerative Medicine. 2013; 8(6): 771-782. Available from: https://doi.org/10.2217/rme.13.71.

[11] Bifari F, Dolci S, Bottani E, et al. Complete neural stem cell (NSC) neuronal differentiation requires a branched 
chain amino acids-induced persistent metabolic shift towards energy metabolism. Pharmacological Research. 2020; 158:104863. Available from: doi:10.1016/j.phrs.2020.104863.

[12] Horikoshi Y, Yan Y, Terashvili M, et al. Fatty acid-treated induced pluripotent stem cell-derived human cardiomyocytes exhibit adult cardiomyocyte-like energy metabolism phenotypes. Cells. 2019; 8(9): 1095. Available from: doi:10.3390/cells8091095.

[13] Frayne E. Conserved mechanism of action of a phosphate analogue in cultured cells. Mol Cell Biochem. 2016; 415: 111-117. Available from: doi:10.1007/s11010-016-2681-6.

[14] Casadesús J, Low D. Epigenetic gene regulation in the bacterial world. Microbiology and molecular biology reviews: $M M B R$. 2006; 70(3): 830-856. Available from: doi:10.1128/MMBR.00016-06.

[15] Soung G, Miller J, Koc H, Koc E. Comprehensive analysis of phosphorylated proteins of Escherichia coli ribosomes. Journal of Proteome Research. 2009; 8(7): 3390-3402. Available from: doi:10.1021/pr900042e.

[16] Potel C, Lin M, Heck A, et al. Widespread bacterial protein histidine phosphorylation revealed by mass spectrometry-based proteomics. Nat Methods. 2018; 15: 187-190. Available from: doi:10.1038/nmeth.4580.

[17] Iyer S, Casas-Pastor D, Kraus D, et al. Transcriptional regulation by $\sigma$ factor phosphorylation in bacteria. Nat Microbiol. 2020; 5: 395-406. Available from: doi:10.1038/s41564-019-0648-6. 